\title{
Shaping of Dynamic Responses by Observers
}

\author{
Chiu H. Choi*
}

Electrical Engineering Program, University of North Florida, Jacksonville, FL 32224, USA

\begin{abstract}
This paper presents the experimental results of the investigation of the effects of the observer poles for shaping the dynamic responses of state-space systems with system poles placed by state vectors obtained from full-order observers instead of the original full state vectors, which is assumed to be unavailable. The particular parameters considered in the shaping of the dynamic responses in this paper are the settling time and percent overshoot. A large number of observer poles on the complex plane were selected evenly. The corresponding observer state vectors were computed. These state vectors were used for system pole placement. All the system poles were placed at the same locations. The resulting dynamic responses were simulated and the settling times and percent overshoots were measured. Even though the system poles were the same, the dynamic responses, in particular, the settling time and percent overshoot were not identical and depended on the locations of the observer poles. The relationship between these parameters and the observer poles were analyzed and the results are presented in this paper.
\end{abstract}

Keywords: Dynamic response shaping, Linear systems, Compensators, Computer-aided control systems design.

\section{INTRODUCTION}

A common technique for shaping the dynamic response of a state space system is by pole placement with full state vector ([1] and [2]). For example, consider the double integrator state space description in [3].

$\dot{x}=\left[\begin{array}{ll}0 & 1 \\ 0 & 1\end{array}\right] x+\left[\begin{array}{l}0 \\ 1\end{array}\right] u$

$y=\left[\begin{array}{ll}1 & 0\end{array}\right] x$

It is desired to stabilize the system and to limit the $2 \%$ settling time to be approximately equal to 4.5 .

Such requirements can be achieved by placing the system poles at locations with real part approximately equal to 0.889 . For example with system poles chosen to be $-0.889 \pm \mathrm{j} 1.056$, the step response of the state space system (1) with full state feedback.

i.e., $u=-K x+r$, is shown in Fig. (1). The settling time is noted to be 4.35 in the figure and roughly meets the specification.

This gain vector $K$ in the input equation $u=-K x+r$ was computed by the "place" command [4] of Matlab and is equal to [1.9055 1.7780]. More accurate settling time can be attained by fine tuning the location of the system poles.

The assumption of no finite zero in the closed-loop transfer function is necessary. It is because finite zeros will contribute to the dynamics of the step response just like the poles but in different manners.

*Address correspondence to this author at the Department of Electrical Engineerng, University of North Florida, 1 UNF Dr. Jacksonville, FL 32224, USA; Tel: (904) 620-1681; E-mail: cchoi@unf.edu

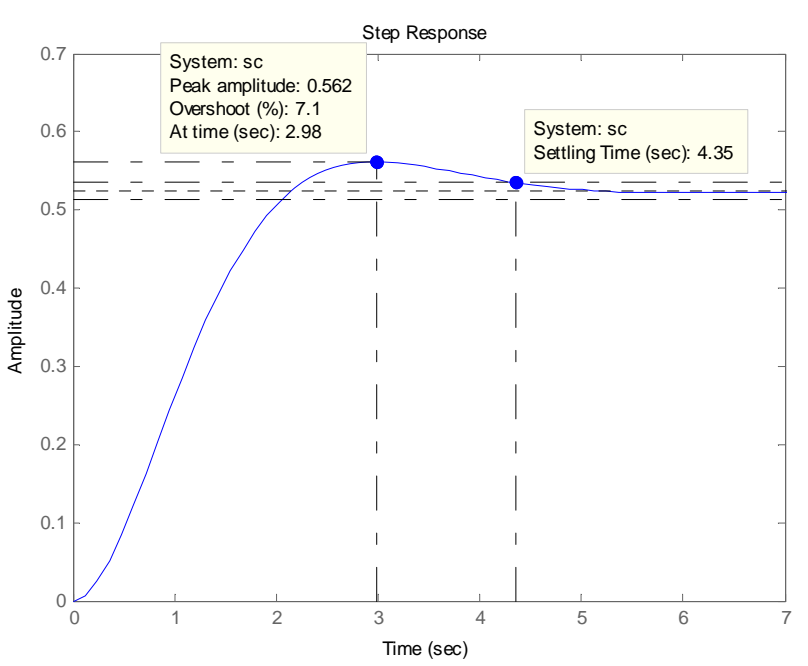

Fig. (1). Step response of equation (1).

If the state vector $x$ in the input $u=-K x+r$ is not available, the full order observer state vector can be used as a substitute $([5,6])$. However, there is an issue which was briefly reported in $[1,7]$. The issue was that the locations of the observer poles could increase or decrease the settling time. The references $[1,7]$ did not provide extensive investigations. In this paper, results of extensive investigations are provided. A deeper study of the effects of the locations of the observer poles on the dynamic responses and the findings are described below.

The contents of the rest of this paper are arranged as follows: the observer approach used in this paper is reviewed in the rest of this section. Section 2 presents the extensive simulation results and the analysis of the effects of the loca- 
tions of the observer poles on the dynamic responses. Section 3 provides concluding remarks.

Generally for a single-input, single-output controllable and observable state-space system

$\dot{x}=A x+B u$

$y=C x$

with full state vector $x$ being available, the input $u$ can be constructed as

$u=r-K x$

such that the eigenvalues of $A-B K$ are placed at the desired locations that meet the dynamic response, in particular, step response performance criteria.

If the state vector $x$ is not available. An observer can be used to construct an estimate ( $\hat{x})$ of the original system state vector. This estimate, which is the observer state vector, can be used in place of the original state vector in the pole placement method for placing the system poles at the desired locations. A summary of this approach follows. Consider a state-space model

$\dot{x}=A x+B u ; \quad x(0)=x_{0}$

$y=C x$

The corresponding observer equation is:

$\dot{\hat{x}}=A \hat{x}+B u+L(y-\hat{y})=(A-L C) \hat{x}+B u+L y ; \hat{x}(0)=\hat{x}_{0}$

$\hat{y}=C \hat{x}$

and the input equation is:

$u=r-K \hat{x}$,

where $K$ is the gain matrix that drives the compensated system to operate at the desired system poles corresponding to the dynamic response requirements, e.g., settling time.

The combined original system equation and the observer equation is

$$
\begin{aligned}
& {\left[\begin{array}{c}
\dot{x} \\
\dot{\hat{x}}
\end{array}\right]=\left[\begin{array}{cc}
A & -B K \\
L C & A-B K-L C
\end{array}\right]\left[\begin{array}{l}
x \\
\hat{x}
\end{array}\right]+\left[\begin{array}{l}
B \\
B
\end{array}\right] r ; \quad\left[\begin{array}{l}
x(0) \\
\hat{x}(0)
\end{array}\right]=\left[\begin{array}{l}
x_{0} \\
\hat{x}_{0}
\end{array}\right]} \\
& y=[\mathrm{C} 0]\left[\begin{array}{l}
x \\
\hat{x}
\end{array}\right]
\end{aligned}
$$

Define the error $e=x-\hat{x}$. The error equation can be obtained as

$\dot{e}=(A-L C) e ; \quad e(0)=x_{0}-\hat{x}_{0}$

If the original system is observable, the poles of the error equation can be place sufficiently far from the imaginary axis and in the left half plane so that the error will die away very quickly. This observer state vector will place the system poles at the desired locations but the resulting step response may not meet the expected requirements such as settling time, peak time, and percent overshoot. The initial conditions $\left(x_{0}\right)$ of the observer and the poles of the observer (which are the eigenvalues of $(A-L C))$ have effects on the step response and can change its shape. This paper presents the results of the effect of the observer poles on the settling time.

\section{SIMULATION RESULTS}

Consider the double integrator system in equation (1). The problem is to stabilize the system and to meet the performance criterion that the $2 \%$ settling time is to be 4.5 . A simple solution is by placing the system poles at the same locations at $-0.889 \pm \mathrm{j} 1.056$, as the example in Section 1 . Assume that the actual state vector $x$ needed in the pole placement was not available. An observer is used for obtaining estimates of the state vector $x$. It is intended to investigate the effect of the observer poles on the settling time. One hundred observers with distinct observer poles were tried. These one hundred distinct pairs of observer poles are

$-0.889 \mathrm{~m} \cdot \cos \left(\frac{\pi(\mathrm{n}-1)}{20}\right) \pm \mathrm{j} \cdot 0.889 \mathrm{~m} \cdot \sin \left(\frac{\pi(\mathrm{n}-1)}{20}\right)$

where $\mathrm{m}=1,2, \ldots, 10$; and $\mathrm{n}=1,2, \ldots, 10$.

A diagram of these one hundred pairs of complex conjugate observer poles is shown in Fig. 2. Note that the variable $m$ indicates the distance of the observer poles from the origin and the variable $n$ indicates the phase of the observer poles. The amplitude of the observer poles in (9) is $0.889 \mathrm{~m}$ and that ranges from 0.889 to 8.89 . The phase of the observer poles is $\left(\pi-\frac{\pi(\mathrm{n}-1)}{20}\right)$ and that ranges from $\pi \mathrm{rad}$ to $\frac{11 \pi}{20} \mathrm{rad}$.

For each pair of complex conjugate observer poles, the corresponding observer state vector was computed. The initial condition of the observer was chosen to be $\hat{x}_{0}=\left[\begin{array}{l}0.1 \\ 0.1\end{array}\right]$.

The observer state vector was used in pole placement for placing the system poles at the locations of $-0.889 \pm j 1.056$. The step response of the resulting system was obtained with the initial condition of the original system (1) chosen as $x_{0}=\left[\begin{array}{l}0.0 \\ 0.0\end{array}\right]$. The settling time of the step response was measured. These procedures were repeated for every pair of the one hundred pairs of complex conjugate observer poles. The settling times of these one hundred cases are shown in Tables $\mathbf{1}$ and $\mathbf{2}$. Note that the radial line of observer poles on the $x$-axis in Fig. (2) produces settling times as shown in column $n=1$ in Table 1 . The green radial line of observer poles immediately above the previous one produces settling times as shown in the column $n=2$ in Table 1 . The rest of the radial lines of observer poles in Fig. (2) produces settling times in the same order as shown in the rest of Table $\mathbf{1}$ and Table 2. In each column of Tables 1 and 2 all the corresponding observer poles have the same phase but the amplitude changes from 0.889 to 8.99 .

Each column of Tables $\mathbf{1}$ and $\mathbf{2}$ is plotted as shown in Fig. (3). A three dimensional plot for Tables $\mathbf{1}$ and $\mathbf{2}$ with settling time vs. $m$ and $n$ is shown in Fig. (4).

In Fig. (3), the legend Ts1 $(1: 10$, i) is the plot of the column in Table $\mathbf{1}$ and Table $\mathbf{2}$ with $\mathrm{n}=\mathrm{i}$. The following observations are noted in Figs. (3 and $\mathbf{4})$.

First, when $\mathrm{m}$ is large enough, i.e., when the distance of the observer poles from the origin is far enough from the 
Table 1. Settling Time for $m$ Between 1 and 10, and $n$ Between 1 and 5

\begin{tabular}{|c|c|c|c|c|c|}
\hline & $\mathbf{n = 1}$ & $\mathbf{2}$ & $\mathbf{3}$ & $\mathbf{4}$ & 3.05 \\
\hline \hline $\mathrm{m}=1$ & 3.15 & 3.14 & 3.10 & 4.56 & 4.69 \\
\hline 2 & 4.55 & 4.55 & 4.56 & 4.35 & 4.55 \\
\hline 3 & 4.38 & 4.38 & 4.37 & 4.27 & 4.32 \\
\hline 4 & 4.30 & 4.30 & 4.29 & 4.24 & 4.22 \\
\hline 5 & 4.26 & 4.25 & 4.25 & 4.20 & 4.21 \\
\hline 6 & 4.23 & 4.23 & 4.22 & 4.20 & 4.19 \\
\hline 7 & 4.21 & 4.21 & 4.20 & 4.19 & 4.19 \\
\hline
\end{tabular}

Table 2. Settling Time for $\mathrm{m}$ Between 1 and 10, and $\mathrm{n}$ Between 6 and 10

\begin{tabular}{|c|c|c|c|c|c|}
\hline & $\mathbf{n = 6}$ & $\mathbf{7}$ & $\mathbf{8}$ & $\mathbf{9}$ & $\mathbf{1 0}$ \\
\hline \hline $\mathrm{m}=1$ & 5.92 & 6.59 & 6.82 & 9.35 & 5.45 \\
\hline 2 & 4.51 & 4.41 & 4.25 & 4.24 & 8.96 \\
\hline 3 & 4.27 & 4.22 & 4.16 & 4.14 & 5.57 \\
\hline 4 & 4.23 & 4.20 & 4.18 & 4.17 & 4.16 \\
\hline 5 & 4.21 & 4.19 & 4.18 & 4.23 & 4.12 \\
\hline 6 & 4.20 & 4.18 & 4.17 & 4.16 & 4.16 \\
\hline 7 & 4.19 & 4.18 & 4.17 & 4.16 & 4.17 \\
\hline 9 & 4.18 & 4.18 & 4.17 & 4.17 & 4.17 \\
\hline
\end{tabular}

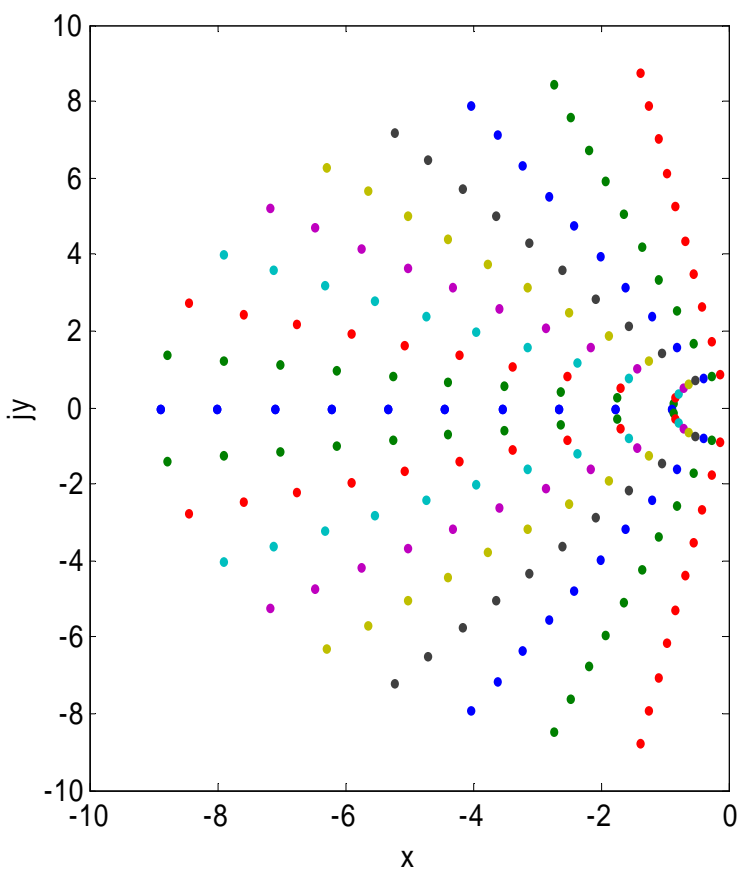

Fig. (2). All the observer poles of equation (9).

desired system poles, the settling time of the step response in the resulting system (7) is fairly constant and depends much less on the phases of the observer poles. In other words when observer poles in the outer band of Fig. (2) are chosen, the corresponding settling times of the resulting systems (7) are about the same, regardless of the phase of the observer poles. 
Table 3. Settling Time for $m$ Between 1 and 10, and $\mathbf{n}$ Between 1 and 5

\begin{tabular}{|c|c|c|c|c|c|}
\hline & $\mathbf{n = 1}$ & $\mathbf{2}$ & $\mathbf{3}$ & $\mathbf{4}$ & $\mathbf{5}$ \\
\hline \hline $\mathrm{m}=1$ & 6.18 & 6.18 & 6.16 & 6.13 & 4.53 \\
\hline 2 & 4.63 & 4.62 & 4.59 & 4.21 & 4.44 \\
\hline 3 & 4.28 & 4.27 & 4.25 & 4.11 & 4.15 \\
\hline 4 & 4.15 & 4.15 & 4.13 & 4.07 & 4.07 \\
\hline 5 & 4.10 & 4.09 & 4.08 & 4.05 & 4.05 \\
\hline 6 & 4.07 & 4.07 & 4.06 & 4.04 & 4.04 \\
\hline 7 & 4.06 & 4.05 & 4.05 & 4.04 & 4.03 \\
\hline 9 & 4.05 & 4.05 & 4.04 & 4.04 & 4.04 \\
\hline
\end{tabular}

Table 4. Settling Time for $\mathrm{m}$ Between 1 and 10, and $\mathrm{n}$ Between 6 and 10

\begin{tabular}{|c|c|c|c|c|c|}
\hline & $\mathbf{n = 6}$ & $\mathbf{7}$ & $\mathbf{8}$ & $\mathbf{9}$ & $\mathbf{1 0}$ \\
\hline \hline $\mathrm{m}=1$ & 5.99 & 8.27 & 9.12 & 9.57 & 5.44 \\
\hline 2 & 5.66 & 5.78 & 5.65 & 4.02 & 8.90 \\
\hline 3 & 4.07 & 3.97 & 3.87 & 3.96 & 3.65 \\
\hline 4 & 4.04 & 4.01 & 3.99 & 4.01 & 4.16 \\
\hline 5 & 4.03 & 4.02 & 4.00 & 4.01 & 4.91 \\
\hline 6 & 4.03 & 4.02 & 4.02 & 4.02 & 4.03 \\
\hline 7 & 4.03 & 4.02 & 4.02 & 4.03 & 4.02 \\
\hline 9 & 4.03 & 4.03 & 4.03 & 4.03 & 4.03 \\
\hline
\end{tabular}

Second, it is known that by pushing the observer poles deeper into the left half plane, the result is that the sooner the observer states converge to the actual system states. But for certain systems such as the example in this section, having the observer states converging faster to the actual system states is not a critical factor in meeting the settling time requirement. Observer poles not being deep into the left half plane can achieve about the same settling time as the deep observer poles as long as their distances from the origin are far enough from the desired system poles.

Third, consider those observer poles that are at the same radial distance as that of the desired system poles to the origin. These observer poles correspond to smaller $m$ 's such as $m=1$. Their corresponding settling times fluctuate much and depends much on the phases of the observer poles. The fluctuation of the settling times decreases as the radial distance increases (i.e., as $\mathrm{m}$ increases).

Fourth, the settling times for $m=5$ or 6 are about equal to those of $m=10$. This indicates that as the observer poles are sufficiently far from the system poles, pushing the observer poles even farther away does not change the settling time that much. Further, note that the settling requirement of 4.5 units were best met by those $\mathrm{m}=2$, i.e., observer poles that are twice as far from the origin as the system poles. Pushing the observer poles deeper into the left half plane will ensure the observer state converging faster to the system state but not necessarily producing a settling time closer to the requirement.
To further investigate the observations above, another extensive set of simulations was performed. In this second set of simulations, the state space model as shown in equation (10) below is considered.

$$
\begin{aligned}
& \dot{x}=\left[\begin{array}{ll}
0 & 1 \\
0 & 1
\end{array}\right] x+\left[\begin{array}{l}
0 \\
1
\end{array}\right] u \\
& y=\left[\begin{array}{ll}
1 & 0
\end{array}\right] x
\end{aligned}
$$

It is an unstable, controllable and observable system. The problem is to stabilize the system and to limit the $2 \%$ settling time to approximately equal to 4.5 . Assume that the actual state vector $x$ needed in the pole placement was not available. An observer is used for obtaining estimates of the system state vector $x$.

It is intended to investigate again the effect of the observer poles on the settling time. Again one hundred observers with distinct observer poles were simulated. The same set of observer poles as shown in Fig. (2) and the same observer initial conditions were used in the simulation of (7) with (10) as the original system. The settling times of these one hundred cases of observer poles are shown in Tables $\mathbf{3}$ and $\mathbf{4}$. Each column of Tables $\mathbf{3}$ and $\mathbf{4}$ is plotted as shown in Fig. (5).

A three dimensional plot for Tables $\mathbf{3}$ and $\mathbf{4}$ with settling time vs. $m$ and $n$ is shown in Fig. (6).

In Fig. (5), the legend Ts2 $(1: 10, i)$ is the plot of the column in either Table 3 or Table 4 with $n=i$. The trend of the 


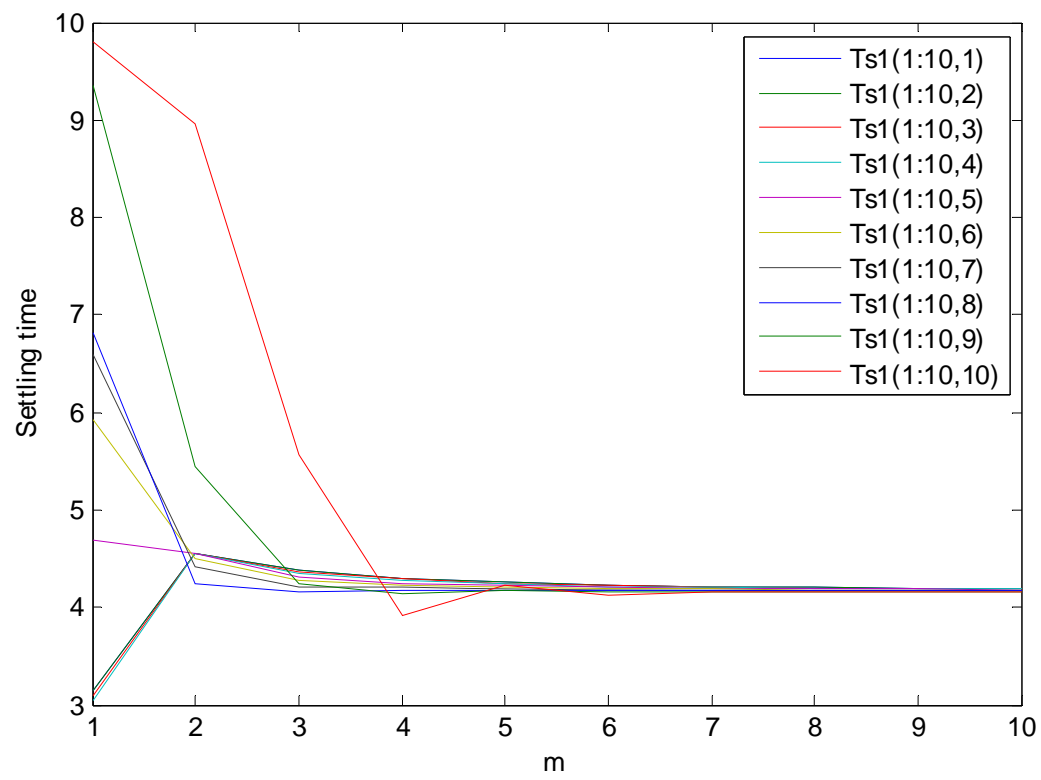

Fig. (3). Settling time vs. $\mathrm{m}$ for $\mathrm{n}$ from 1 to 10 .

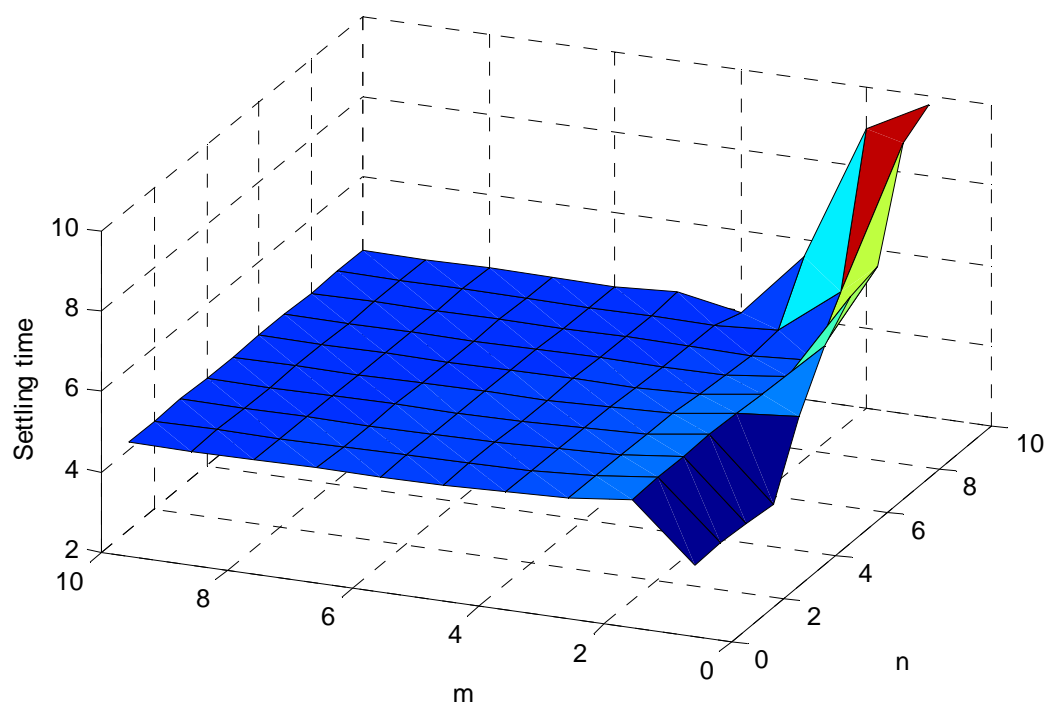

Fig. (4). plot of the settling time vs. $m$ and $n$.

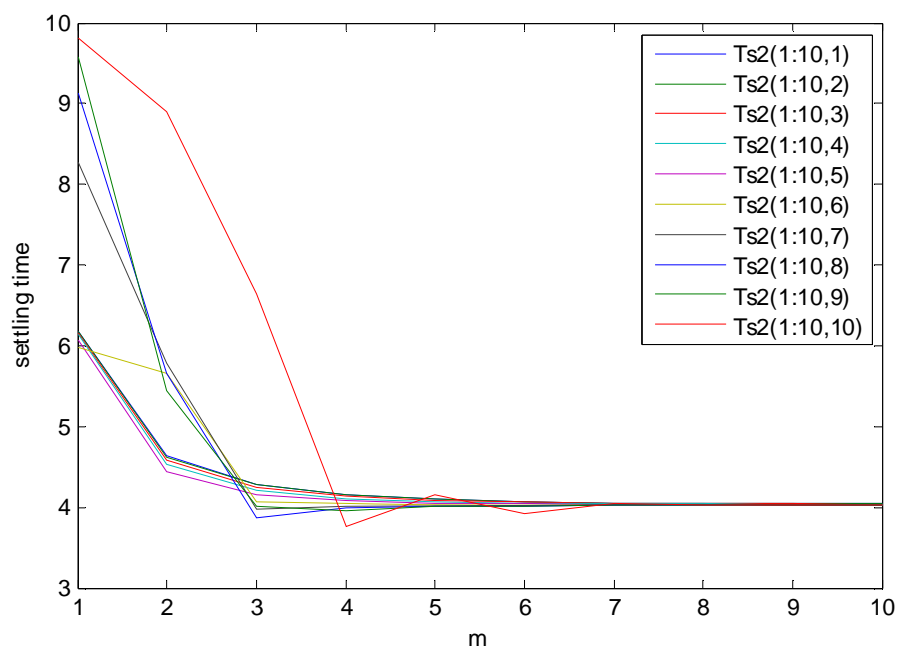

Fig. (5). Settling time vs. $\mathrm{m}$ for $\mathrm{n}$ from 1 to 10 . 


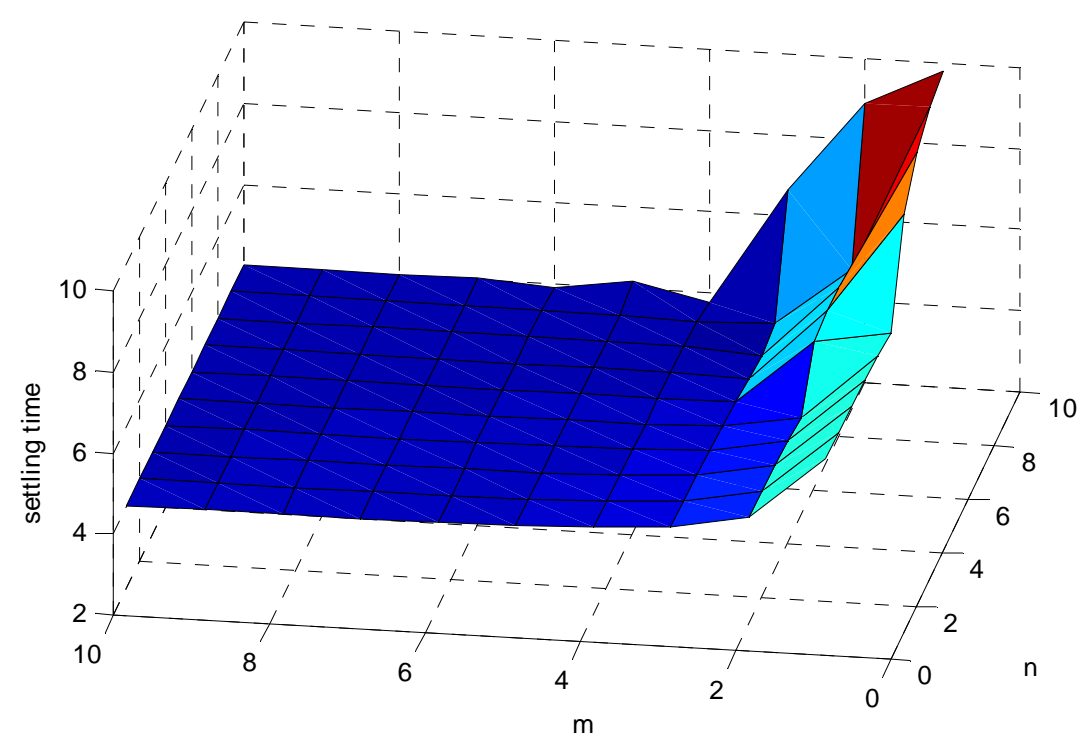

Fig. (6). Plot of the settling time vs. $m$ and $n$.

lines in Fig. (5) is similar to those of Fig. (3). The trend of the surface plot in Fig. (6) is similar to that of Fig. (4). The same observations as in the previous set of observations are noticed again in this new set of simulations.

\section{CONCLUDING REMARKS}

The examples presented in this paper indicate that the observer poles can change the shape of the system step response. The effects of the magnitudes and the phases of the observer poles were investigated. For dynamic response requirement such as settling time, it does not depend much on the phase of observer poles when they are sufficiently far away from the system poles as indicated in the simulations in the previous section. When the observer poles are close to the system poles, the resulting settling time depends on the phase of the observer poles. The approach presented in this paper can also be applied in the future investigation of the effects of the observer poles on other dynamic response requirements such as peak time, rise time, and percent overshoot.

\section{CONFLICT OF INTEREST}

The authors confirm that this article content has no conflicts of interest.

\section{ACKNOWLEDGEMENT}

Declared none.

\section{REFERENCES}

[1] N.S. Nise, Control Systems Engineering, Wiley: NJ, 2004.

[2] R.L. Williams II, and D.A. Lawrence, Linear State-space Control Systems Theory and Design, Wiley: NJ, 2007.

[3] C.H. Choi, "Effect of Observer Poles on Step Responses," Proc. IEEE Southeast Symposium on System Theory, 2010; pp. 122-126.

[4] J. Kautsky, and N.K. Nichols, "Robust pole assignment in linear state feedback," International Journal of Control, vol. 41, pp. 1129-1155, 1985.

[5] L.A. Zadeh and C.A. Desoer, Linear System Theory: The State Space Approach, McGraw-Hill: NY, 1963.

[6] B. Friedland, Control Systems Design, McGraw-Hill: NY, 1986.

[7] C.H. Choi, "Step Response Improvement by Pole Placement with Observers," In: Proc. IEEE Southeast Symposium on System Theory, 2008; pp. 7-12.

Received: April 18, 2012

(C) Chiu H. Choi; Licensee Bentham Open.

This is an open access article licensed under the terms of the Creative Commons Attribution Non-Commercial License (http://creativecommons.org/licenses/by-nc/3.0/) which permits unrestricted, non-commercial use, distribution and reproduction in any medium, provided the work is properly cited. 